\title{
Differences in Occupational Burnout Among Primary Care Professionals
}

\author{
Jessica Clifton, PhD, Levi Bonnell, MPH, Juvena Hitt, MPH, Abigail Crocker, PhD, \\ Gail L. Rose, PhD, Constance van Eeghen, DrPH, Rodger Kessler, PhD, \\ Kari A. Stephens, PhD, Kathryn Teng, MD, Janeen Leon, MS, RDN, LD, \\ Brenda Mollis, MPA, MPH, and Benjamin Littenberg, MD
}

Background: Occupational burnout is a major concern for personal well-being and patient care. We examined burnout among primary care providers (PCPs), medical residents, behavioral health providers (BHPs), nurses, and other clinical and nonclinical primary care team members.

Methods: This was a cross-sectional study, nested within a larger randomized trial. Participants completed a validated 9-item burnout measure with 3 domains: depersonalization, emotional exhaustion, and personal accomplishment. Multivariable multilevel linear regression with a random intercept for each practice was used to determine mean differences in burnout across professional roles.

Results: Overall burnout rates varied by professional role: PCPs $70 \%$, medical residents $89 \%$, BHPs $59 \%$, nurses $66 \%$, other clinicians $68 \%$, and nonclinical professionals $70 \%$. Compared with nonclinical professionals, residents experienced more burnout in more domains, followed by PCPs. PCPs, residents, and nurses reported significantly worse depersonalization and exhaustion scores. Nonclinical professionals had worse accomplishment scores than all clinical professionals except for residents. This study revealed moderate-to-high levels of burnout among primary care professionals.

Discussion: Clinicians may be experiencing aspects of burnout more intensely than their nonclinical colleagues, and this may be most true for residents and PCPs. Based on these variations, interventions to mitigate burnout may need to be tailored by professional role. ( $\mathrm{J}$ Am Board Fam Med

2021;34:1203-1211.)

Keywords: Cross-Sectional Studies, Depersonalization, Health Personnel, Occupational Burnout, Primary Health Care

\section{Introduction}

Occupational burnout among health care professionals is a major concern for individual well-being and patient care. Specifically, burnout has been associated with lower work satisfaction, greater

This article was externally peer reviewed.

Submitted 1 April 2021; revised 19 July 2021; accepted 21 July 2021.

From University of Vermont, Burlington (JC, LB, JH, AC, GLR, CvE, BL); University of Colorado, Aurora (RK); University of Washington, Seattle (KAS, BM); MetroHealth System, Case Western Reserve University, Cleveland (KT, JL).

Funding: Research reported in this manuscript was funded through a Patient-Centered Outcomes Research Institute (PCORI) Award (PCS-1409-24372). The views, statements, and opinions presented in this manuscript are solely the responsibility of the author(s) and do not necessarily represent the views of PCORI, its Board of Governors or Methodology Committee. PCORI is an independent, nonprofit organization authorized by Congress in 2010. Its mission is to fund research that will provide emotional distress, reduced productivity, and high job turnover, and it can impact quality of care (ie, perceived safety, medical errors) and patient satisfaction. . $^{1,2}$

Burnout results from unrelenting and unmitigated organizational stressors in the workplace. ${ }^{3-5}$ Although conceptualizations of burnout have varied, the bulk of empirical evidence suggests that burnout presents as multiple distinct patterns: emotional exhaustion (ie, feelings of energy depletion), depersonalization (ie, distance or negativity toward

patients, their caregivers, and clinicians with the evidencebased information needed to make better-informed health care decisions. PCORI is committed to continually seeking input from a broad range of stakeholders to guide its work.

Conflict of interest: None.

Corresponding author: Jessica Clifton, $\mathrm{PhD}, 89$ Beaumont Ave S459, Burlington, VT 05405 (E-mail: jessica.clifton@ med.uvm.edu). 
others and work), and diminished personal accomplishment (ie, ineffectiveness at work). ${ }^{6,7}$

Studies of primary care professionals undifferentiated by job role have reported overall burnout rates ranging from $6 \%$ to $89 \%$. However, robust studies comparing burnout between the various roles in a primary care setting are few. ${ }^{8-14}$ Research comparing primary care providers (PCPs) to nonPCPs suggests that burnout may differ by role. ${ }^{9,10,13,15}$ When comparing PCPs with nurses, the results are mixed, with some studies revealing little difference $\mathrm{e}^{12,15}$ and others suggesting that physicians have higher rates of burnout. ${ }^{11,12}$ For instance, a descriptive study examining burnout across multiple roles within Veteran Affairs (VA) primary care settings reported that PCPs (45\%), nurse care managers ( $40 \%$ ), and administrative staff (30\%) had similar rates of overall burnout and that medical assistants and licensed practical nurses had lower burnout rates. ${ }^{12}$ Other descriptive studies have also suggested higher levels of burnout in at least 1 domain among resident physicians compared with nonresident providers and/or staff. ${ }^{15-17}$

Simple prevalence rates do not tell the whole story. More sophisticated analyses can allow us to tease apart other important variables potentially related to both burnout and role. Unfortunately, studies using robust statistical techniques to examine differences among primary care professionals' expressions of burnout are sparse, and these findings are mixed. For instance, 1 study with a moderate sample size $(n=467)$ comparing providers, clinical assistants, and other staff using chi-square testing reported no statistically significant differences in overall burnout. ${ }^{18} \mathrm{~A}$ study that recruited a large sample of primary care professionals and deployed logistic regression models to account for clustered data and confounding variables (ie, work hours, practice size, type of specialty practice, located in underserved areas) revealed that physicians were 1.75 times more likely than staff to report overall burnout. ${ }^{19}$

It is challenging to generalize from these studies given variation in settings (VA only and/or clinics in a specific region/city), measures (emotional exhaustion domain only or general burnout question), statistical techniques (descriptive analysis, univariate analysis, multivariable analysis), and job roles (combining distinctly separate roles under 1 category) to get the full scope of burnout expression by role in primary care. Few if any studies have used more robust statistical techniques that include confounders to examine differences in burnout expression across the domains among primary care professionals. Understanding these discrepancies may supply role-specific avenues for preventing and mitigating burnout. The purpose of this study was to better understand the variations in burnout domains (emotional exhaustion, depersonalization, and personal accomplishment) among primary care professionals across the United States.

\section{Methods}

This study is part of a larger cluster-randomized pragmatic clinical trial, Integrated Behavioral Health and Primary Care for Comorbid Behavioral and Medical Problems (IBH-PC). As part of IBHPC, 45 primary care practices of diverse structures (privately owned, academic, hospital or health system, federally qualified health center), each having an onsite behavioral health provider (BHP), were recruited from diverse geographic regions (urban/ rural) in 13 US states. ${ }^{20}$ The IBH-PC trial sought to test the effectiveness of a practice-level change process designed to enhance integrated behavioral health and improve the health of patients with multiple medical conditions. ${ }^{20}$

An estimated 1100 practice members received at least 1 e-mail invitation from a practice delegate (ie, practice manager, behavioral health or medical director, etc.) to complete the online anonymous survey at 1 time point. This convenience sample of participants completed the survey between June 2018 and October 2019 (before the COVID-19 pandemic). Study procedures were first approved by the University of Vermont Committees on Human Research in 2017. All participants provided informed consent. No compensation was provided to respondents.

\section{Measures}

The anonymous survey incorporated demographic questions along with a validated burnout questionnaire. Demographic questions included age range, gender, race, ethnicity, education, number of years in current occupation, and professional role. Professional options included PCP, medical resident, BHP (psychologists, social workers, and counselors), nurse, and other clinical and nonclinical professionals. Other clinical professionals 
primarily identified as medical assistants and care/ referral coordinators. Many nonclinical participants had administrative, management, or operational roles. We identified medical residents if they met all of the following criteria: (1) endorsed being a PCP with a medical degree, (2) worked in a training clinic, and (3) had less than 3 years of work experience.

An adaption of the Maslach Burnout Inventory (aMBI) was used to assess degree of occupational burnout. $^{21-23}$ The aMBI is a 9 -item questionnaire, where each question is on a 6-point Likert scale $(0=$ never to $5=$ everyday $)$. This measurement is made up of 3 domain scores: Emotional Exhaustion, Depersonalization, and Personal Accomplishment. Example items include: "feel emotionally drained from my work," "become more callous toward people at work," "feel exhilarated working with patients."

The primary outcomes were the 3 domain-specific scores, which we calculated domain scores by summing the item scores for the respective domain. Higher scores for both Emotional Exhaustion (range 0 to 18 ) and Depersonalization (range 0 to 18) subscales indicate greater occupational burnout, whereas lower scores on Personal Accomplishment (range 0 to 18 ) subscale indicate more burnout. We dichotomized domain-specific scores to capture moderate-to-high burnout, defined as a score of 4 or above for depersonalization, 7 or above for emotional exhaustion, and under 14 for accomplishment. ${ }^{24}$ Using this scoring algorithm, we defined Burnout Count as the number of domains on which participants had moderate-to-high burnout scores, ranging from 0 to 3. Total Burnout was defined as the percentage of participants with a burnout count of 1 or higher.

Potential covariates included personal-, practice-, and county-level factors. The anonymous survey included personal variables (ie, age, gender, race, ethnicity, education, and years working in the field). Previous research has reported that these variables can influence burnout. 6,25,26 Practice variables, collected as part of the larger IBH-PC study, included setting (academic, hospital, community health center, private), nonprofit status, specialty (family medicine, internal medicine, mixed), provider full-time equivalents, tenure of behavioral health services, degree of behavioral health integration, training of medical residents, panel size, number of patient encounters, and proportion of Medicare patients. Publicly available county-level variables were matched on the location of the practice. These included region, urban/rural status, population density, social deprivation, ${ }^{27}$ age, sex, race, ethnicity, income, and level of education.

\section{Data Analysis}

Chi-square and Kruskal-Wallace tests were performed to identify group differences for total burnout, burnout count, and domain-specific scores between professional roles. We used multilevel linear regressions to assess the relationships of each domain to professional role. Each role was dummy coded and included in each model. Practice was included as a random intercept to account for the correlation of personal-level measures within the practice they work. Thirty-five potential covariates were identified. If the potential covariate changed the coefficient of any professional role on burnout by more than $\pm 10 \%$ in a model containing only professional role as the predictor, it was included in the final model. Post hoc multiple comparisons were conducted to better understand differences in burnout among occupational roles. aMBI subscales that were missing 1 or more items were removed from the related analyses. Fifty-two participants were missing at least $1 \mathrm{aMBI}$ item. All tests were 2-tailed and $\alpha$ was set to $P<.05$ as the statistical threshold for significance. All analyses were conducted using STATA $16 .^{28}$

\section{Results}

Health care professionals from 41 of 45 of the IBH-PC practices participated. An estimated 63\% ( $n=687)$ of practice members consented to participate in the study. On average, 7 participants (range $=2$ to 44 ) from each practice completed the anonymous survey. Over half of participants identified as non-Hispanic White women, under 45 years of age, with more than 10 years of experience in their occupation. Nearly half of participants completed graduate school. PCPs made up $24 \%$ of the sample. See Table 1.

Sixty-nine percent of all respondents showed evidence of moderate-to-high burnout in at least 1 domain. Total burnout rates were seen across all roles: $70 \%$ of PCPs, $89 \%$ residents, $59 \%$ of $\mathrm{BHPs}$, $66 \%$ nurses, $68 \%$ other clinical, and $70 \%$ nonclinical professionals. These rates significantly differed 
Table 1. Descriptive Statistics of Demographic Characteristics Collected Between 2018 and 2019 of Primary Care Professionals $(\mathrm{n}=687)$

\begin{tabular}{|c|c|c|c|c|c|c|c|}
\hline Characteristics & $\begin{array}{l}\text { Primary Care } \\
\text { Provider }\end{array}$ & Resident & $\begin{array}{c}\text { Behavioral } \\
\text { Health Provider }\end{array}$ & Nurse & $\begin{array}{l}\text { Other Clinical } \\
\text { Staff }\end{array}$ & $\begin{array}{l}\text { Nonclinical } \\
\text { Staff }\end{array}$ & All \\
\hline $\mathrm{n}$ & 167 & 56 & 80 & 122 & 108 & 152 & 687 \\
\hline \multicolumn{8}{|l|}{ Age (years), \% } \\
\hline under 25 & 0.0 & 0.0 & 1.3 & 5.7 & 10.2 & 10.5 & 5.1 \\
\hline 25 to 34 & 30.9 & 85.7 & 28.8 & 23.0 & 37.0 & 24.3 & 28.8 \\
\hline 35 to 44 & 24.2 & 12.5 & 36.3 & 29.5 & 28.7 & 23.0 & 27.0 \\
\hline 45 to 54 & 18.4 & 1.8 & 18.8 & 18.9 & 13.9 & 21.1 & 18.4 \\
\hline 55 to 64 & 20.2 & 0.0 & 12.5 & 18.9 & 10.2 & 18.4 & 17.1 \\
\hline $65+$ & 6.3 & 0.0 & 2.5 & 4.1 & 0.0 & 2.6 & 3.7 \\
\hline Gender (female), \% & 65.5 & 57.1 & 81.0 & 90.9 & 87.8 & 91.3 & 81.0 \\
\hline Race (White), \% & 81.4 & 73.2 & 90.8 & 67.2 & 66.7 & 67.9 & 74.8 \\
\hline Hispanic/Latino, \% & 5.5 & 3.6 & 5.2 & 14.1 & 18.6 & 26.4 & 13.6 \\
\hline $\begin{array}{l}\text { Education (graduate } \\
\text { school), \% }\end{array}$ & 92.4 & 100 & 97.5 & 12.9 & 10.2 & 7.9 & 47.0 \\
\hline $\begin{array}{l}\text { Years in occupation, mean } \\
\text { (standard deviation) }\end{array}$ & $13.0(12.1)$ & $1.9(0.9)$ & $7.9(8.2)$ & $12.4(12.2)$ & $8.0(7.92)$ & $7.9(8.6)$ & $10.4(10.7)$ \\
\hline
\end{tabular}

by role $(P=.014)$. Kruskal-Wallis tests revealed significant differences between job role and the burnout domains: depersonalization $(H(5)=23.45, P \leq$ $.001)$, exhaustion $(H(5)=22.73, \quad P \leq .001)$, and diminished accomplishment $(H(5)=37.05, \quad P \leq$ .001). Furthermore, differences between job role and the number of burnout domains significantly differed $(H(5)=30.39, P \leq .001)$. A third of the sample had a burnout count of 0 , indicating no evidence of burnout, whereas $12 \%$ met burnout criteria for all 3 domains. See Table 2 and Figure 1.

Unadjusted analyses revealed significant differences by role for the continuous and dichotomized burnout domain scores (see Table 3). Multilevel linear regression analyses provided further evidence that the aMBI subscales differed by role (see Table 4). Of the 35 potential covariates tested, only race, education, age, years working in the field, and practice training site status altered the association between role and burnout by more than $10 \%$ and were included in the final models.

Adjusting for the 5 confounders reduced the strength of association for all outcomes. This difference was statistically notable when comparing residents' exhaustion scores to other professionals. Although the unadjusted model results suggested

Table 2. Comparison Tests of Burnout Rates Collected Between 2018 and 2019 by Professional Role

\begin{tabular}{|c|c|c|c|c|c|c|c|c|}
\hline & $\begin{array}{l}\text { Primary } \\
\text { Care } \\
\text { Provider }\end{array}$ & Resident & $\begin{array}{l}\text { Behavioral } \\
\text { Health } \\
\text { Provider }\end{array}$ & Nurse & $\begin{array}{c}\text { Other } \\
\text { Clinical Staff }\end{array}$ & $\begin{array}{l}\text { Nonclinical } \\
\text { Staff }\end{array}$ & All & $P$ \\
\hline $\begin{array}{l}\text { Total burnout } \\
\text { (1 or more } \\
\text { domains) }\end{array}$ & $70 \%$ & $89 \%$ & $59 \%$ & $66 \%$ & $68 \%$ & $70 \%$ & $69 \%$ & $0.014^{*}$ \\
\hline $\begin{array}{c}\text { Burnout } \\
\text { count }\end{array}$ & & & & & & & & $<0.001^{\dagger}$ \\
\hline 0 domains & $30 \%$ & $11 \%$ & $41 \%$ & $34 \%$ & $32 \%$ & $31 \%$ & $31 \%$ & \\
\hline 1 domain & $38 \%$ & $26 \%$ & $34 \%$ & $28 \%$ & $39 \%$ & $43 \%$ & $36 \%$ & \\
\hline 2 domains & $24 \%$ & $24 \%$ & $17 \%$ & $25 \%$ & $20 \%$ & $18 \%$ & $21 \%$ & \\
\hline 3 domains & $8 \%$ & $39 \%$ & $8 \%$ & $13 \%$ & $10 \%$ & $9 \%$ & $12 \%$ & \\
\hline
\end{tabular}

Note: Depersonalization, $\mathrm{n}=633$; Emotional Exhaustion, $\mathrm{n}=685$; Personal Accomplishment, $\mathrm{n}=634$.

*Chi-square test was performed comparing groups for dichotomous severity domain scores.

${ }^{\dagger}$ Kruskal-Wallis test was performed comparing groups for continuous domain scores. 
Figure 1. Boxplot of burnout domain scores collected between 2018 and 2019 by professional role.
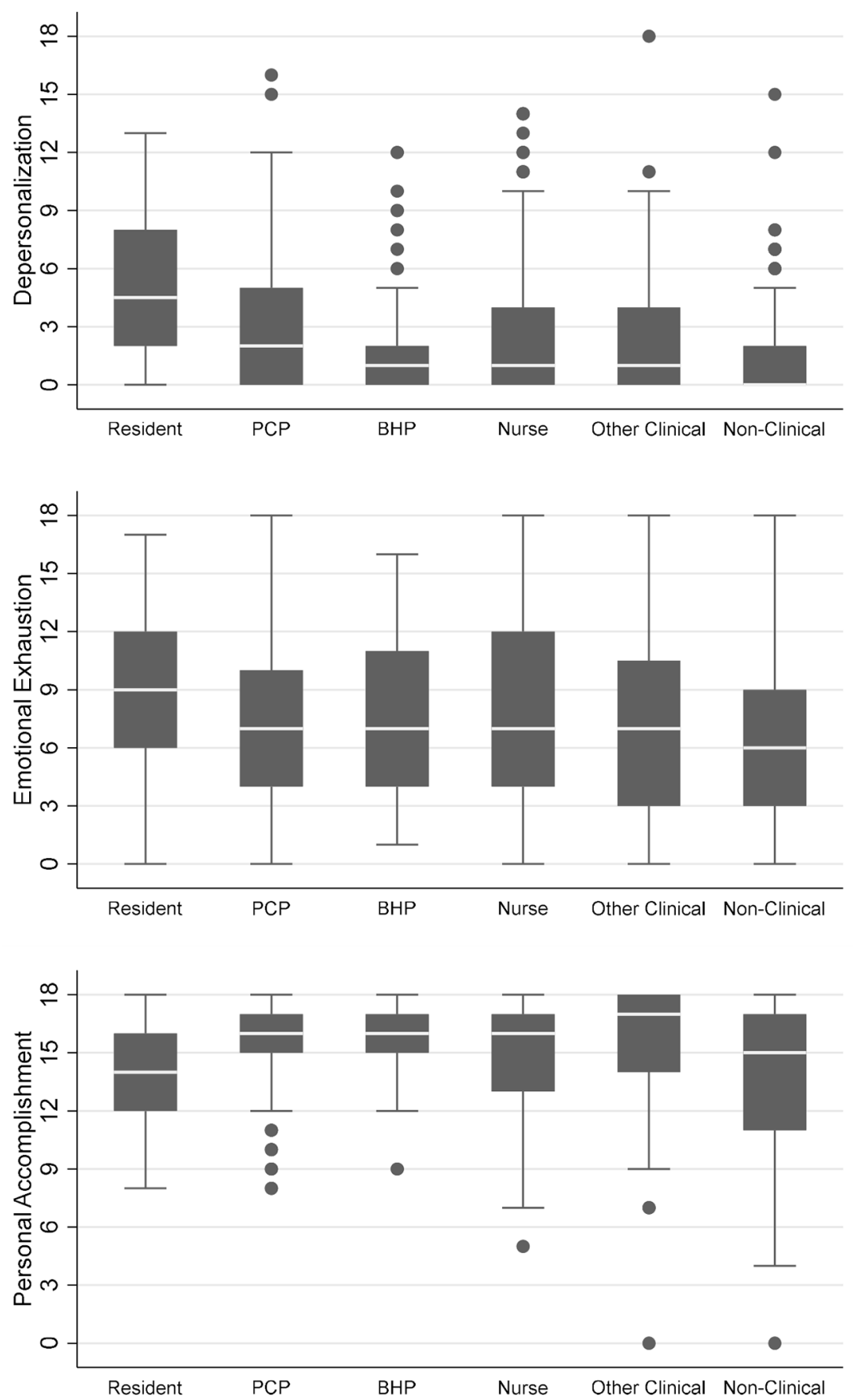

Notes: $\mathrm{PCP}=$ Primary care provider; $\mathrm{BHP}=$ Behavioral Health Provider. Scores range from 0-18. Higher scores for both the Depersonalization $(n=633)$ and Emotional Exhaustion $(n=685)$ domains indicate worse burnout whereas lower scores on the Accomplishment $(n=634)$ domain are worse. 
that residents had higher exhaustion scores than other roles $(B=-2.96$ to -1.56 points; $P<.02)$, the adjusted model revealed no significant differences among clinical roles (see Table 4).

Medical residents had significantly higher depersonalization scores than other job roles $(B=1.36$ to 2.98; $P \leq .02)$ and lower Personal Accomplishment subscale scores than other clinical roles $(B=-1.93$ to -1.21 points; $P \leq .022)$. PCPs reported higher levels of depersonalization than other professional roles, aside from medical residents. Nonclinical professionals had lower burnout scores across the domains compared with many other clinical professionals. See Table 4 for adjusted multilevel results.

\section{Discussion}

Most primary care professionals experienced occupational burnout. The level of burnout measured in this sample is comparable to rates found in other studies. ${ }^{8,9,11-13,15,29-32}$ Strikingly, a third of professionals experienced moderate-to-severe burnout in more than 1 domain, which is higher than previous reports. ${ }^{33}$ These findings further emphasize that burnout for most professionals working in health care is the rule rather than the exception. Furthermore, the intensity of burnout varied by professional role.

Regardless of working in the same setting, these findings suggest that the expression of burnout varies by role. Similarities in the burnout profiles of PCPs and nurses may be explained by the similarities of their job. Primary care nurses, physicians, and advanced practitioners overlap in job roles and responsibilities. ${ }^{34}$ Although BHPs and other clinical professionals have direct involvement in patient care, they may experience less depersonalization than PCPs and nurses due to differences in operational demands and time pressures. For instance, BHPs and other clinical professionals may have more flexibility around documentation, scheduling, and time spent with a patient than PCPs and nurses. Similarly, nonclinical professionals may be experiencing the least amount of exhaustion and depersonalization because they are not involved in direct patient care and thereby do not have the same responsibilities, liabilities, and job duties. In other words, the logistic and emotional demands of direct patient care may explain why clinicians experience higher rates of emotional exhaustion and 
Table 4. Multivariate Regression Analyses of Burnout Domains Scores Collected 2018 to 2019 by Professional Role with Covariates

\begin{tabular}{|c|c|c|c|c|c|c|}
\hline & \multicolumn{2}{|c|}{ Depersonalization } & \multicolumn{2}{|c|}{ Exhaustion } & \multicolumn{2}{|c|}{ Accomplishment } \\
\hline & $B$ & $95 \%$ CI & $B$ & $95 \% \mathrm{CI}$ & $B$ & $95 \%$ CI \\
\hline Resident (base case) & 0.00 & - & 0.00 & - & 0.00 & - \\
\hline PCP & $-1.36^{*}$ & $-2.47,-0.26$ & -0.60 & $-2.11,0.91$ & $1.21^{*}$ & $0.18,2.25$ \\
\hline BHP & $-2.73^{*}$ & $-3.89,-1.56$ & -0.99 & $-2.59,0.62$ & $1.49^{*}$ & $0.41,2.58$ \\
\hline Nurse & $-1.80^{*}$ & $-3.24,-0.36$ & -0.47 & $-2.37,1.43$ & $1.47^{*}$ & $0.13,2.81$ \\
\hline Other clinical & $-2.59^{*}$ & $-4.03,-1.16$ & -1.20 & $-3.11,0.71$ & $1.93^{*}$ & $0.61,3.26$ \\
\hline Nonclinical & $-2.98^{*}$ & $-4.42,-1.54$ & $-2.11^{*}$ & $-4.02,-0.20$ & 0.17 & $-1.16,1.51$ \\
\hline Constant (intercept) & $4.13^{*}$ & $-2.33,5.94$ & $7.85^{*}$ & $5.40,10.31$ & $14.56^{*}$ & $12.87,16.23$ \\
\hline \multicolumn{7}{|l|}{ Comparison } \\
\hline PCP vs BHP & $-1.36^{*}$ & $-2.26,-0.47$ & -0.39 & $-1.61,0.83$ & 0.28 & $-0.55,1.11$ \\
\hline vs Other clinical & $-1.23^{*}$ & $-2.37,-0.08$ & -0.60 & $-2.09,0.89$ & 0.72 & $-0.34,1.78$ \\
\hline vs Nonclinical & $-1.62^{*}$ & $-2.73,-0.50$ & $-1.52^{*}$ & $-3.00,-0.07$ & $-1.04^{*}$ & $-2.07,-0.01$ \\
\hline Nonclinical vs BHP & 0.26 & $-1.01,1.52$ & 1.13 & $-0.54,2.80$ & $1.32^{*}$ & $0.15,2.49$ \\
\hline vs Nurse & $1.18^{*}$ & $0.37,2.00$ & $1.64^{*}$ & $0.56,2.73$ & $1.29^{*}$ & $0.54,2.05$ \\
\hline vs Other clinical & 0.39 & $-0.43,1.21$ & 0.91 & $-0.20,2.03$ & $1.76^{*}$ & $1.00,2.52$ \\
\hline
\end{tabular}

CI, confidence interval.

Note: Multivariable regression analyses with practice-level clustering and covariates (race, graduate school, age, years working, training status). Depersonalization, $\mathrm{n}=633$; Emotional Exhaustion, $\mathrm{n}=685$; Personal Accomplishment, $\mathrm{n}=634 . B=$ unstandardized independent regression coefficient when accounting for all the other variables in the model.

$\mathrm{BHP}$, behavioral health provider; $\mathrm{PCP}$, primary care provider.

${ }^{*}=P<.05$.

depersonalization as compared with their nonclinical counterparts.

Nonclinical professionals are likely to be younger and have less work experience and education compared with clinicians. These factors have been related to feelings of diminished accomplishment. ${ }^{35,36}$ Furthermore, medicine has a clear hierarchy where large discrepancies exist in both tangible (eg, pay) and nontangible (eg, respect) aspects of the work environment between clinicians and nonclinicians. ${ }^{36-38}$ These differences may explain why nonclinical professionals experience diminished accomplishment.

This study has notable limitations. It used a cross-sectional design with a convenience sample from only 13 states, which can bias the results and limit the ability to draw conclusions. However, given the diversity of practices (ie, structure, size, urban/rural) from the 4 Census regions (West, Midwest, South, Northeast), ${ }^{39}$ it is likely that the results are reasonably representative of colocated primary care practices across the nation. Future research examining the differences and interaction of burnout rates among primary care team members in colocated and non-colocated practices across the nation is warranted. As with all cross- sectional analyses, confounding by unmeasured or unknown factors may introduce error.

This study expands on our knowledge of variations in burnout by role. By exploring burnout expression in diverse primary care settings across the nation and including multiple covariates, we were able to explore variations in professionals' burnout experiences while accounting for other known factors related to burnout. These findings suggest that medical professionals (PCPs and nurses), nonmedical clinicians, and nonclinical professionals differ in burnout expression and intensity. These distinctions in role and burnout expression may explain the contradictory findings of previous research.

Clinicians, those responsible for direct patient care, may be suffering from burnout more intensely than their nonclinical colleagues. This may be worse for PCPs, who are often described as the "quarterback" of the team. ${ }^{40}$ Furthermore, PCPs' socialization and norms may differ substantially from other roles (eg, "detached concern," "learning how to not feel"). ${ }^{41,42}$ Medical residents have the added burdens of high expectations of performance in high-pressure conditions, minimal autonomy, a steep learning curve, inconsistent supervision, long 
work hours, low wages, and high debt. ${ }^{43,44}$ Similarly, differences in the backgrounds and roles of nonclinicians may lead to a diminished sense of personal accomplishment. Since burnout varies by job role, interventions tailored by role that target change at the organizational, leader, and group level may mitigate burnout.

The authors thank Niranjana Das, Maggie Evans, Martin Seehuus, Abby Young, Garyn Worrall, Jesse Reinking, Jen O'Rourke-Lavoie, Christine Runyan, Rachel Postupack; UVM's Clinical Research Outcomes Workshop group; IBHPC coinvestigators, collaborators, and stakeholders; and Living $\&$ Working in Healthcare participants.

To see this article online, please go to: http://jabfm.org/content/ 34/6/1203.full.

\section{References}

1. Patel RS, Bachu R, Adikey A, Malik M, Shah M. Factors related to physician burnout and its consequences: a review. Behav Sci (Basel) 2018;8:98.

2. Salyers MP, Bonfils KA, Luther L, et al. The relationship between professional burnout and quality and safety in healthcare: a meta-analysis. J Gen Intern Med 2017;32:475-82.

3. ICD-11 for Mortality and Morbidity Statistics. QD85 Burnout; 2020. Available from: https://icd. who.int/browse11/l-m/en\#/http://id.who.int/icd/ entity/129180281. Accessed February 1, 2021.

4. Adler D. Burnout: old wine in new bottles. Psychiatr Serv 2020;71:217-8.

5. Maslach C, Schaufeli WB, Leiter MP. Job burnout. Annu Rev Psychol 2001;52:397-422.

6. Purvanova RK, Muros JP. Gender differences in burnout: a meta-analysis. J Vocat Behav 2010;77: $168-85$.

7. Maslach C, Leiter MP. Understanding the burnout experience: recent research and its implications for psychiatry. World Psychiatry 2016;15:103-11.

8. Bruhl EJ, MacLaughlin KL, Allen SV, et al. Association of primary care team composition and clinician burnout in a primary care practice network. Mayo Clin Proc Innov Qual Outcomes 2020;4:135-42.

9. Grumbach K, Knox M, Huang B, Hammer H, Kivlahan C, Willard-Grace R. A longitudinal study of trends in burnout during primary care transformation. Ann Fam Med 2019;17:S9-S16.

10. Willard-Grace R, Knox M, Huang B, Hammer H, Kivlahan C, Grumbach K. Burnout and health care workforce turnover. Ann Fam Med 2019;17:36-41.

11. Edwards ST, Marino M, Balasubramanian BA, et al. Burnout among physicians, advanced practice clinicians and staff in smaller primary care practices. J Gen Intern Med 2018;33:2138-46.
12. Helfrich CD, Dolan ED, Simonetti J, et al. Elements of team-based care in a patient-centered medical home are associated with lower burnout among VA primary care employees. J Gen Intern Med 2014;29 Suppl 2:S659-66.

13. Nocon RS, Fairchild PC, Gao Y, et al. Provider and staff morale, job satisfaction, and burnout over a 4year medical home intervention. J Gen Intern Med 2019;34:952-9.

14. Khanna N, Montgomery R, Klyushnenkova E. Joy in work for clinicians and staff: identifying remedial predictors of burnout from the Mini $Z$ survey. J Am Board Fam Med 2020;33:357-67.

15. Willard-Grace R, Hessler D, Rogers E, Dubé K, Bodenheimer T, Grumbach K. Team structure and culture are associated with lower burnout in primary care. J Am Board Fam Med 2014;27: 229-38.

16. West CP, Dyrbye LN, Sloan JA, Shanafelt TD. Single item measures of emotional exhaustion and depersonalization are useful for assessing burnout in medical professionals. J Gen Intern Med 2009;24:1318-21.

17. Dyrbye LN, West CP, Satele D, et al. Burnout among US medical students, residents, and early career physicians relative to the general US population. Acad Med 2014;89:443-51.

18. Spinelli WM, Fernstrom KM, Galos DL, Britt HR. Extending our understanding of burnout and its associated factors: providers and staff in primary care clinics. Eval Health Prof 2016;39:282-98.

19. Goldberg DG, Soylu TG, Grady VM, Kitsantas P, Grady JD, Nichols LM. Indicators of workplace burnout among physicians, advanced practice clinicians, and staff in small to medium-sized primary care practices. J Am Board Fam Med 2020;33: 378-85.

20. Crocker AM, Kessler R, van Eeghen C, et al. Integrating Behavioral Health and Primary Care (IBH-PC) to improve patient-centered outcomes in adults with multiple chronic medical and behavioral health conditions: study protocol for a pragmatic cluster-randomized control trial. Trials 2021;22: 200-18.

21. Riley MR, Mohr DC, Waddimba AC. The reliability and validity of three-item screening measures for burnout: evidence from group-employed health care practitioners in upstate New York. Stress Heal 2018;34:187-93.

22. McManus IC, Winder BC, Gordon D. The causal links between stress and burnout in a longitudinal study of UK doctors. Lancet 2002;359:2089-90.

23. McManus IC, Gordon D, Winder BC. Duties of a doctor: UK doctors and good medical practice. BMJ Qual Saf 2000;9:14-22.

24. Thimmapuram JR, Grim R, Bell T, et al. Factors influencing work-life balance in physicians and advance practice clinicians and the effect of 
heartfulness meditation conference on burnout. Glob Adv Health Med 2019;8.

25. Gómez-Urquiza JL, Vargas C, De la Fuente EI, et al. Age as a risk factor for burnout syndrome in nursing professionals: a meta-analytic study. Res Nurs Health 2017;40:99-110.

26. Lawrence JA, Davis BA, Corbette T, Hill EV, Williams DR, Reede JY. Racial/ethnic differences in burnout: a systematic review. J Racial Ethn Heal Disparities 2021. Available from: https://link. springer.com/article/10.1007\%2Fs40615-020-00950-0.

27. Butler DC, Petterson S, Phillips RL, Bazemore AW. Measures of social deprivation that predict health care access and need within a rational area of primary care service delivery. Health Serv Res 2013;48:539-59.

28. StataCorp S. Data Analysis and Statistical Software. Version 16.0. 2018..

29. Rotenstein LS, Torre M, Ramos MA, et al. Prevalence of burnout among physicians: a systematic review. JAMA Netw Open 2018;320:1131-50.

30. Monsalve-Reyes CS, San Luis-Costas C, GómezUrquiza JL, Albendín-García L, Aguayo R, Cañadas-De la Fuente GA. Burnout syndrome and its prevalence in primary care nursing: a systematic review and meta-analysis. BMC Fam Pract 2018; 19:59.

31. Morse G, Salyers MP, Rollins AL, Monroe-DeVita M, Pfahler C. Burnout in mental health services: a review of the problem and its remediation. Adm Policy Ment Health 2012;39:341-52.

32. García-Sierra R, Fernández-Castro J, MartínezZaragoza F. Relationship between job demand and burnout in nurses: does it depend on work engagement? J Nurs Manag 2016;24:780-8.

33. Soler JK, Yaman H, Esteva M, European General Practice Research Network Burnout Study Group, , et al. Burnout in European family doctors: the EGPRN study. Fam Pract 2008;25:245-65.

34. Page N. Task overlap among primary care team members: an opportunity for system redesign? J Healthc Manag 2006;51:295.
35. Lim N, Kim EK, Kim H, Yang E, Lee SM. Individual and work-related factors influencing burnout of mental health professionals: a metaanalysis. J Employ Couns 2010;47:86-96.

36. Yektatalab S, Honarmandnejad K, Janghorban R. Relationship between occupational burnout and demographic variables among nurses in Jahrom, Iran. Pan Afr Med J 2019;34:22.

37. US Bureau of Labor Statistics. Occupational employment and wages, May 2017; 2018. Available from: https://www.bls.gov/oes/2017/may/oes291062. htm. Accessed February 1, 2021.

38. Leape LL, Shore MF, Dienstag JL, et al. Perspective: a culture of respect, part 1: the nature and causes of disrespectful behavior by physicians. Acad Med 2012;87:845-52.

39. US Census. 2010 Census region and divisions of the United States; 2010. Available from: https:// www.census.gov/geographies/reference-maps/2010/ geo/2010-census-regions-and-divisions-of-theunited-states.html. Accessed June 20, 2021.

40. Gray MF, Sweeney J, Nickel W, et al. Function of the medical team quarterback: patient, family, and physician perspectives on team care coordination in patient-and family-centered primary care. Perm J 2019;23. Available from: https://www.thepermanente journal.org/issues/2019/fall/7228-primary-care.html.

41. Angoff NR. Making a place for emotions in Medicine. Yale J Heal Policy Law Ethics 2013; 2:8.

42. Underman K, Hirshfield LE. Detached concern? Emotional socialization in twenty-first century medical education. Soc Sci Med 2016;160:94101.

43. Rodrigues H, Cobucci R, Oliveira A, et al. Burnout syndrome among medical residents: a systematic review and meta-analysis. PLoS One 2018;13: e0206840.

44. Golob A, Beste LA, Stern M, Johnson K. Emotional distress among physician residents and fellows: an observational study of trainees seeking counseling visits. Acad Psychiatry 2018;42:25-30. 